\title{
ESTUDO COMPARATIVO ENTRE UM PROGRAMA DE EXERCÍCIO FÍSICO AERÓBIO E UM TREINAMENTO DE MEMÓRIA EPISÓDICA EM INDIVÍDUOS IDOSOS $^{i}$
}

\author{
Gisele de Paula Vieira Membro do Grupo de Estudo sobre Doença de \\ Parkinson (GEDOPA), Universidade Federal do Rio \\ de Janeiro, Rio de Janeiro, Brasil. Mestre. Docente do \\ curso de Fisioterapia das Faculdades de Ciências \\ Médicas e da Saúde de Juiz de Fora - SUPREMA Juiz \\ de Fora, Minas Gerais, Brasil.
}

\author{
Vernon Furtado da Silva Doutor. Laboratório de Neuromotricidade e \\ Performance Motora (LANPED) Instituto de \\ Educação Física e Desportos, Universidade do Estado \\ do Rio de Janeiro, Rio de Janeiro, Rio de Janeiro, \\ Brasil.
}

\section{Clynton Lourenço Correa}

Membro do Grupo de Estudo sobre Doença de Parkinson (GEDOPA), Universidade Federal do Rio de Janeiro, Rio de Janeiro, Brasil. Doutor, Departamento de Fisioterapia, Universidade Federal do Rio de Janeiro, Rio de Janeiro, Brasil.

Endereço para correspondência: giselefisio04@hotmail.com

\begin{abstract}
Resumo
Introdução: $\mathrm{O}$ avanço da idade repercute em declínio cognitivo, mas continua sendo um fenômeno heterogêneo. $\mathrm{Na}$ verdade, vários fatores extrínsecos parecem modular o impacto do envelhecimento sobre a cognição. Objetivo: Neste contexto, o presente estudo teve como objetivo, estudar os possíveis efeitos do treinamento de memória e do exercício aeróbio em idosos com queixa de déficit de memória. Método: Foi realizado um estudo experimental, cuja amostra foi composta por um montante de 30 idosos saudáveis, de ambos os gêneros, residentes na cidade de Cataguases - MG, com idades entre 60 a 70 anos, divididos em três grupos. Grupo 1, realizou exercício físico aeróbio (GEF); o grupo 2, realizou um treinamento de memória utilizando a técnica de categorização (GTM) e o grupo 3, grupo controle (GC) não realizou nenhuma intervenção. Resultados: No pósteste tanto o GTM quanto o GEF obtiveram melhora no teste de figuras recordadas, contudo o GTM alcançou melhor desempenho na recordação de figuras, apresentando significância estatística na comparação pré e pósteste. Conclusão: Conclui-se com o presente estudo que o treinamento de memória episódica para idosos saudáveis promove melhorias da habilidade mnemônica, assim como o exercício aeróbio contribui também para potencialização de tal habilidade.
\end{abstract}

Palavras-chave: Envelhecimento; Exercício aeróbico; Memória de curto prazo.

\section{COMPARATIVE STUDY BETWEEN AEROBIC EXERCISE AND MEMORY TRAINING IN ELDERLY SUBJECTS}

\begin{abstract}
Introduction: Advancing age reflect on cognitive decline, but remains a heterogeneous phenomenon. In fact, several extrinsic factors seem to modulate the impact of aging on cognition. Objectives: In this context, this study aimed to study the possible effects of memory and aerobic exercise training in elderly subjects with complaints of memory deficit. Methods: It was carried out an experimental study, whose sample was composed of a total of 30 healthy elderly individuals of both genders, citizens from Cataguases - MG, aged between 60 and 70 years, divided into three groups. Group 1 performed aerobic exercise (EG) group 2, performed a memory training using the technique of categorization (MTG), and group 3, control group (CG) did not undergo any intervention. Results: In the post-test both groups, the EG and MTG, showed improvement of the test figures 
remembered, however, the MTG has achieved better recall of pictures, showing a statistical significance when comparing pre-and post-test. Conclusion: In conclusion, the present study shows that the episodic memory training for healthy older adults promotes improvements in mnemonic ability, as well as aerobic exercise contributes also for this ability.

Keywords: Aging; Aerobic Exercise; Short-term memory.

\section{INTRODUÇÃO}

O envelhecimento é um processo dinâmico, progressivo e fisiológico, acompanhado por modificações morfo-funcionais, assim como modificações bioquímicas e psicológicas, resultando na diminuição da reserva funcional dos órgãos e aparelhos. ${ }^{(1)}$ De acordo com projeções da Organização Mundial de Saúde, entre o período de 1960 e 2025, o grupo de idosos no Brasil deverá crescer quinze vezes enquanto a população total crescerá cinco vezes. Dessa forma, o Brasil ocupará o $6^{\circ}$ lugar quanto ao contingente de idosos, com cerca de 32 milhões de pessoas com 60 anos ou mais e, em breve, teremos pirâmides etárias semelhantes às dos países europeus. ${ }^{(2)}$ Tais transformações trazem desafios para todos os setores, impondo a necessidade de repensar a dimensão da oferta de serviços de saúde necessários para as próximas décadas para essa população. ${ }^{(3)}$

Os idosos apresentam maior vulnerabilidade a doenças e uma parcela significativa é acometida por alterações cognitivas esperadas para a faixa etária. ${ }^{(4)}$ Essas alterações variam em sua forma e intensidade de indivíduo para indivíduo, devido a diferenças no estilo de vida, condições de saúde, alimentação, prática de atividades físicas, entre outros fatores. ${ }^{(5)}$ Alterações esperadas no envelhecimento incluem diminuição na memória operacional (manutenção e processamento simultâneos) ${ }^{(6)}$ e na memória episódica (eventos específicos). ${ }^{(7,8)} \mathrm{O}$ treino cognitivo e de memória no idoso saudável pode ser capaz de aproximar seu desempenho atual do seu desempenho máximo possível, revelando a existência de plasticidade cognitiva, pois os idosos têm capacidade de aprender e empregar estratégias mnemônicas de forma mais eficaz. ${ }^{(9)}$

A atividade física, considerada uma ferramenta de baixo custo, é um dos fatores mais importantes e acessíveis para prevenir e proteger as funções cerebrais. ${ }^{(10)} \mathrm{O}$ treinamento aeróbio pode melhorar consideravelmente aspectos críticos do sistema de atenção, o controle executivo, e a atividade do córtex pré-frontal e regiões parietais ${ }^{(1)}$ Assim, a aptidão cardiovascular pode promover um aumento significativo da eficiência, capacidade de adaptação, e plasticidade do cérebro, e, por conseguinte, pode reduzir a senescência biológica e cognitiva. ${ }^{(12,13)}$ Nesse sentido, o presente artigo tem como objetivo comparar os possíveis 
efeitos do treinamento de memória e do exercício aeróbio em idosos com queixa de déficit de memória.

\section{METODOLOGIA}

O presente estudo foi experimental, tipo longitudinal, com ênfase no desempenho da memória em idosos saudáveis em dois momentos distintos pré e pós-treinamento comparando os resultados intra e inter-grupos. Este estudo foi aprovado pelo Comitê de Ética em Pesquisa (COMEP) da Universidade Castelo Branco, Protocolo 0153/2008. A amostra foi caracterizada por 30 indivíduos idosos de ambos os gêneros, residentes na cidade de Cataguases, Minas Gerais, com idade entre 60 a 70 anos, subdivididos aleatoriamente em três grupos, sendo cada grupo composto por 10 indivíduos. O grupo 1 realizou exercício físico aeróbio (GEF), o grupo 2, realizou um treinamento de memória utilizando a técnica de categorização (GTM), e o grupo 3 (controle controle - GC) não realizou nenhuma intervenção. Os voluntários não poderiam apresentar distúrbios auditivo, visual, físico ou mental. Todos pertencentes a uma mesma classe social, nível de escolaridade e hábitos diários, dentre os quais não se incluía prática de atividade física, logo, indivíduos sedentários. A seleção ocorreu através de uma divulgação por Folder, sobre uma Oficina de Memória. Todos os participantes eram voluntários, portanto, foi solicitada a concordância, por escrito, dos mesmos como condição para participação no projeto.

Inicialmente foram apresentados aos grupos os objetivos e métodos da pesquisa e solicitada à concordância de forma voluntária de cada indivíduo, através de preenchimento de um termo de consentimento livre e esclarecido.

Foi utilizada a Escala de Depressão Geriátrica - (EDG-15) ${ }^{(14)}$ que é uma versão curta da escala original ${ }^{(15)}$ e o Mini - Exame do Estado Mental - MEEM $^{(16)}$ para detecção de comprometimento cognitivo dos participantes. Após a seleção, os voluntários realizaram a avaliação de memória episódica ${ }^{(17)}$ que consta de índices de nomeação, familiaridade e complexidade, para fins de coleta de dados pré e pós-intervenção. Para tanto, os indivíduos receberam um protocolo com as instruções necessárias à realização da tarefa de memória episódica, a prancha de figuras, uma tarefa de códigos, uma pergunta para responderem o máximo de figuras que estava contida na prancha, uma pergunta discursiva e oito objetivas sobre as estratégias utilizadas para memorizar as figuras. Tal protocolo foi lido pela pesquisadora juntamente com os participantes para maior esclarecimento da tarefa. 
Os participantes do GEF realizaram 8 sessões, durante 1 mês, 2 vezes por semana com duração de 50 minutos cada. Os 10 minutos iniciais foram direcionados ao aquecimento e os 10 minutos finais ao desaquecimento muscular como medidas de prevenção de lesões músculo-esqueléticas. Os 30 minutos restantes foram direcionados à prática em bicicleta ergométrica horizontal (Movement ${ }^{\circledR}$ ), nível de carga 0,5 a $3 \mathrm{Kgfm}$. A aferição da pressão arterial foi realizada antes, durante e após a execução da atividade aeróbica, tendo como critério de exclusão indivíduos com freqüência cardíaca de repouso superior a 120 bpm; pressão arterial sistólica superior a $180 \mathrm{mmHg}$ e pressão arterial diastólica superior a 100 $\mathrm{mmHg}^{(18)}$ e a resistência mínima utilizada foi de $0,5 \mathrm{Kg}$. Já os participantes do GTM, também realizaram o treinamento no período de 1 mês, sendo 2 encontros semanais com duração de 50 minutos cada, totalizando 8 sessões destinadas ao treino de memória utilizando a técnica de categorização, em sala apropriada para execução do treinamento por meio do teste SKT, Short Cognitive Performance Test, já validado para a população brasileira. ${ }^{(19,20)}$ Cada prancha continha 12 figuras, e foram categorizadas em sub-grupos como automóveis, objetos de cozinha, entre outros. ${ }^{(8)}$ Durante as sessões de treinamento foram apresentados aos participantes o conceito de categorização e ensinados a utilizar a estratégia em todas as sessões estimulando o uso da mesma por meio de material visual e verbal alternadamente. Os participantes do GC realizaram o pré e pós-testes na mesma semana dos grupos experimentais e receberam uma versão abreviada do treinamento na semana após as avaliações de pós-teste.

Os procedimentos estatísticos empregados no estudo foram de natureza descritiva e inferencial, sendo analisadas tanto as relações intergrupos quanto as intragrupos. As ferramentas utilizadas para as respectivas análises estavam presentes no pacote estatístico SSPS 12.0, sendo adotada a seguinte ordem de análise: Inicialmente para uma comparação intergrupos, e para determinar a natureza paramétrica ou não paramétrica dos dados, aplicouse o teste de normalidade de "Kolmogorov-Smirnov" em todas as variáveis do estudo, caso essa análise apontasse para os dados como paramétricos, adotar-se-ia a análise de variância ONEWAY ANOVA e como teste complementar, caso necessário, o teste de TUKEY. Para as comparações intragrupos, dos momentos pré e pós-teste, optou-se pelo teste t de STUDENT. Em todas as análises adotou-se o índice relevância de $p<0,05$ para sua aceitação.

\section{RESULTADOS}

A descrição dos resultados das variáveis observadas nos três grupos que compuseram a amostra da pesquisa está exposta na tabela 1. Na análise descritiva dos testes EDG-15 e MEEM, as médias dos grupos ficaram dentro dos padrões de normalidade. Com relação ao 
teste de Velocidade de Processamento Mental, em média, o GTM conseguiu a maior porcentagem de transferência tanto no momento pré-teste comparado ao GC $(+0,08 \%)$ e GEF $(+0,08 \%)$ quanto no pós-teste comparado ao GC $(+0,07 \%)$ e GEF $(+0,08 \%)$. O GTM obteve o melhor resultado na análise descritiva pré e pós-teste.

Com relação ao teste Figuras Recordadas o GEF obteve melhor desempenho nesta tarefa tanto no pré como no pós-testes, seguidos pelo GTM, como apresentado na tabela 1.

Tabela 1 - Apresentação descritiva do número total de indivíduos, média dos escores, desvio padrão e limites, superior e inferior, dos testes aplicados nos três grupos que formaram o estudo.

\begin{tabular}{|c|c|c|c|c|c|c|}
\hline TESTES & GRUPOS & $\mathrm{N}$ & $\begin{array}{c}\text { MÉDIA DOS } \\
\text { ESCORES }\end{array}$ & $\begin{array}{l}\text { DESVIO } \\
\text { PADRÃO }\end{array}$ & MÍNIMO & MÁXIMO \\
\hline \multirow{3}{*}{$\begin{array}{c}\text { ESCALA DE } \\
\text { DEPRESSÃO } \\
\text { GERIÁTRICA (EDG) }\end{array}$} & CONTROLE & 10 & 2,30 & 1,33 & 0,00 & 4,00 \\
\hline & $\begin{array}{l}\text { TREINO DE } \\
\text { MEMÓRIA }\end{array}$ & 10 & 2,10 & 1,28 & 0,00 & 4,00 \\
\hline & $\begin{array}{c}\text { EXERCÍCIO } \\
\text { FÍSICO } \\
\end{array}$ & 10 & 1,80 & 1,54 & 0,00 & 4,00 \\
\hline \multirow{3}{*}{$\begin{array}{l}\text { MINI EXAME DO } \\
\text { ESTADO MENTAL } \\
\text { (MEEM) }\end{array}$} & CONTROLE & 10 & 28,80 & 0,78 & 28,00 & 30,00 \\
\hline & $\begin{array}{l}\text { TREINO DE } \\
\text { MEMÓRIA }\end{array}$ & 10 & 29,20 & 0,42 & 29,00 & 30,00 \\
\hline & $\begin{array}{c}\text { EXERCÍCIO } \\
\text { FÍSICO }\end{array}$ & 10 & 28,30 & 1,25 & 26,00 & 30,00 \\
\hline \multirow{3}{*}{$\begin{array}{l}\text { VELOCIDADE DE } \\
\text { PROCESSAMENTO } \\
\text { MENTAL PRÉ-TESTE } \\
\text { (VPM-PRÉ) }\end{array}$} & CONTROLE & 10 & 20,47 & 8,87 & 10,70 & 35,70 \\
\hline & $\begin{array}{l}\text { TREINO DE } \\
\text { MEMÓRIA }\end{array}$ & 10 & 23,96 & 11,98 & 10,70 & 45,00 \\
\hline & $\begin{array}{l}\text { EXERCÍCIO } \\
\text { FÍSICO }\end{array}$ & 10 & 21,06 & 7,27 & 11,42 & 33,57 \\
\hline \multirow{3}{*}{$\begin{array}{l}\text { VELOCIDADE DE } \\
\text { PROCESSAMENTO } \\
\text { MENTAL PÓS-TESTE } \\
\text { (VPM-PÓS) }\end{array}$} & CONTROLE & 10 & 19,81 & 7,62 & 8,57 & 32,10 \\
\hline & $\begin{array}{l}\text { TREINO DE } \\
\text { MEMÓRIA }\end{array}$ & 10 & 25,66 & 11,34 & 14,20 & 46,40 \\
\hline & $\begin{array}{c}\text { EXERCÍCIO } \\
\text { FÍSICO }\end{array}$ & 10 & 22,28 & 7,16 & 10,71 & 34,28 \\
\hline \multirow{3}{*}{$\begin{array}{c}\text { FIGURAS } \\
\text { RECORDADAS PRÉ- } \\
\text { TESTE } \\
\text { (FR-PRÉ) }\end{array}$} & CONTROLE & 10 & 25,80 & 6,01 & 19,40 & 36,10 \\
\hline & $\begin{array}{l}\text { TREINO DE } \\
\text { MEMÓRIA }\end{array}$ & 10 & 30,23 & 9,19 & 19,40 & 44,40 \\
\hline & $\begin{array}{c}\text { EXERCÍCIO } \\
\text { FÍSICO }\end{array}$ & 10 & 57,74 & 16,71 & 36,10 & 86,10 \\
\hline \multirow{3}{*}{$\begin{array}{c}\text { FIGURAS } \\
\text { RECORDADAS PÓS- } \\
\text { TESTE. } \\
\text { (FR-PÓS) }\end{array}$} & CONTROLE & 10 & 23,31 & 5,26 & 16,60 & 33,30 \\
\hline & $\begin{array}{l}\text { TREINO DE } \\
\text { MEMÓRIA }\end{array}$ & 10 & 40,24 & 6,94 & 30,50 & 52,70 \\
\hline & $\begin{array}{l}\text { EXERCÍCIO } \\
\text { FÍSICO }\end{array}$ & 10 & 75,83 & 21,35 & 50,00 & 100,00 \\
\hline
\end{tabular}


$\mathrm{Na}$ aplicação do teste de normalidade de "Kolmogorov-Smirnov", os índices encontrados em todas as análises foram superiores a 0,05, caracterizando assim, natureza paramétrica de todos os dados da pesquisa, e credenciando a utilização da ANOVA ONEWAY como teste de análise de variância. Os dados referentes a essas análises estão dispostos na tabela 2 .

Tabela 2 - Apresentação dos graus de liberdade, valores de F e índice de significância das análises de variância aplicadas nos dados de todos os testes que compuseram o estudo.

\begin{tabular}{|c|c|c|c|c|}
\hline Testes aplicados & gl (1) & gl (2) & $\mathrm{F}$ & Valor $\mathrm{p}$ \\
\hline EDG & & & 0,325 & 0,725 \\
\hline MEEM & & & 2,577 & 0,095 \\
\hline VPM-PRÉ & & & 0,380 & 0,688 \\
\hline & 2 & 27 & & \\
\hline VPM-PÓS & & & 1,088 & 0,351 \\
\hline FR-PRÉ & & & 22,459 & $0,000^{*}$ \\
\hline FR-PÓS & & & 40,504 & $0,000^{*}$ \\
\hline
\end{tabular}

* índice de significância p<0,05. Legenda: gl: graus de liberdade, EDG - Escala de Depressão Geriátrica, MEEM - Mini - Exame do Estado Mental, VPM - Velocidade de processamento mental e FR - Figuras recordadas.

Na tabela 2 nota-se que os escores de figuras recordadas, nos momentos pré e pósteste, apresentaram índice na análise de variância $\mathrm{p}<0,05$, demonstrando que existiram diferenças significativas na comparação intra-grupos para esses dados. Assim, aplicou-se o teste de Tukey para determinar onde estavam essas diferenças.

As múltiplas comparações feitas pelo teste de Tukey mostraram que no teste de Figuras Recordadas, no momento pré-teste, os índices $\mathrm{p}<0,05$, foram encontrados apenas nas comparações entre o GC e o GEF, e entre o GTM e GEF. Já para o momento pós-teste desse mesmo teste, as comparações entre o GTM com o GC e o GEF mostraram diferença estatisticamente significativa $(\mathrm{p}<0,05)$.

A figura 1 apresenta os dados que indicam que apenas a intervenção aplicada no GTM, no Teste Figuras Recordadas, apresentou significância estatística na comparação pré e pós-teste. Contudo, cabe ressaltar que essa significância estatística ficou próxima de ser alcançada no GEF, nesse mesmo teste, onde é possível perceber que os escores relativos à avaliação da velocidade de processamento mental referente às três coletas foram bem 
próximos quando evidenciamos os grupos que sofreram intervenção. No GC ocorreu uma discreta involução nos escores desta avaliação.

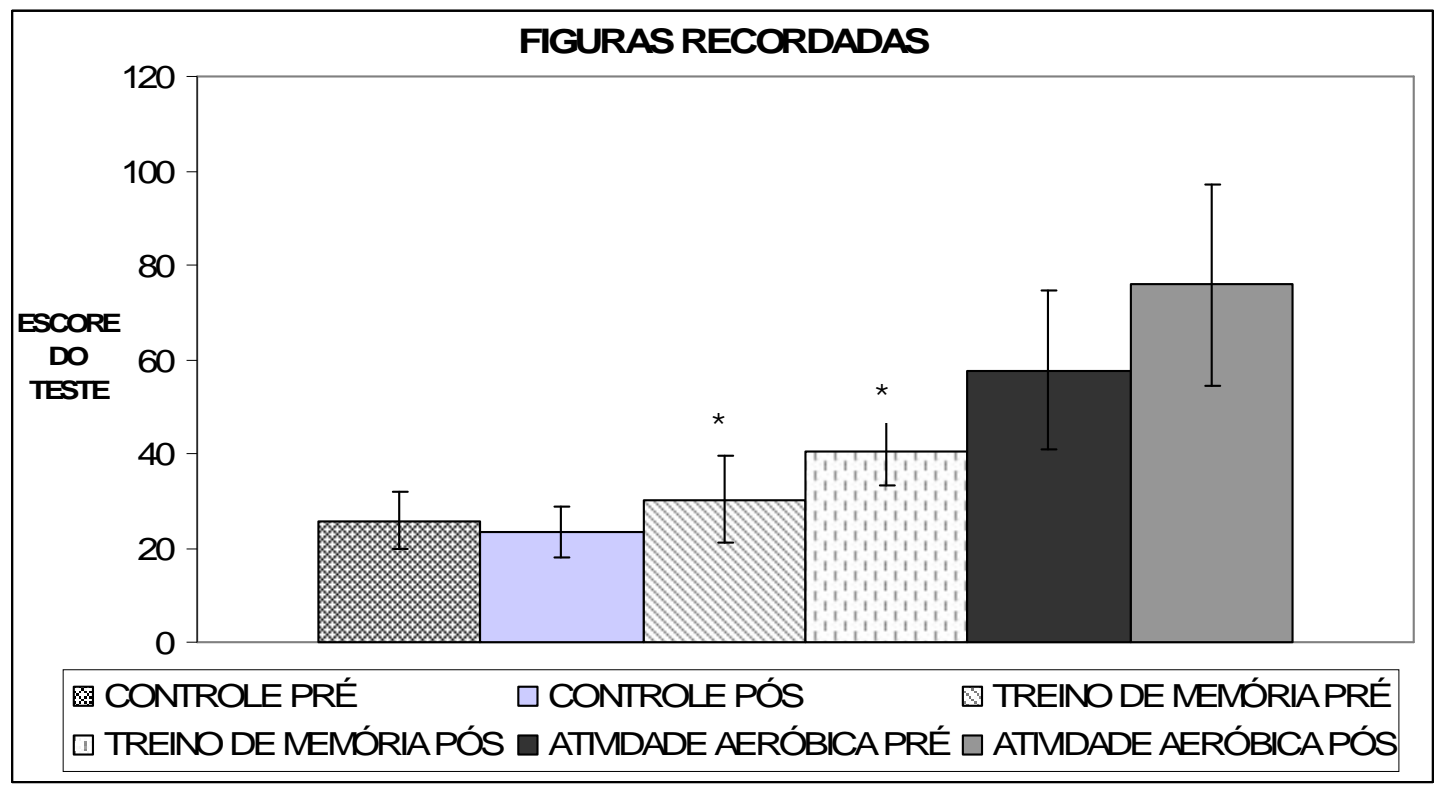

Figura 1 -Média dos escores do teste Figuras Recordadas nos momentos pré e pós-intervenção, no grupo controle, grupo treino de memória e atividade aeróbica. * índice de significância $\mathrm{p}<0,05$.

\section{DISCUSSÃO}

Um estudo ${ }^{(21)}$ atribui ao treinamento de memória episódica para idosos saudáveis a melhora significativa nas tarefas treinadas. Em outro estudo, autores compararam os efeitos entre o treinamento físico aeróbio e treinamento na função cognitiva. ${ }^{(20)}$ Os autores alocaram os indivíduos em quatro grupos (treinamento aeróbio, treinamento mental, combinando treinamento aeróbio e mental e controle). O programa de exercícios aeróbios, e o programa de treinamento mental alcançaram o mesmo grau de melhora da função cognitiva (memória), sugerindo a hipótese que a utilização conjugada dos dois métodos pode otimizar os resultados devido a melhora no coeficiente de memória que foi maior do que utilizando apenas uma técnica específica, e o grupo controle não apresentou alteração nas variáveis fisiológicas nem cognitivas. Contudo, a falta de comparação dos resultados alcançados com um grupo que não tenha participado de qualquer tipo de atividade, impõe limites no sentido de conclusões definitivas. Os resultados do presente estudo indicam que os idosos não acometidos por demências, podem apresentar benefícios utilizando essas intervenções já que as comparações foram realizadas entre três grupos, sendo que o grupo controle não participou de nenhuma 
intervenção e foi apenas avaliado e reavaliado nos mesmo espaço de tempo que os demais grupos.

Estudos apontam, ainda, elevação da auto-estima e do bem-estar em indivíduos que fazem exercícios regularmente. ${ }^{(22,23,24)}$ Isso ocorre por consequência do grande número de relatos que evidenciam a melhora do humor, da ansiedade, e da depressão e a resistência a doenças, além de evitar e/ou minimizar o nível de estresse com a prática de exercícios. ${ }^{(25)}$

Estudos epidemiológicos apontam que pessoas moderadamente ativas minimizam o risco de serem acometidas por disfunções mentais quando comparadas com pessoas sedentárias, demonstrando dessa forma, que o ingresso em programas de exercício físico exerce benefícios tanto para habilidades físicas como para as funções cognitivas. ${ }^{(26)}$

Baseado em estudos prévios, buscou-se aperfeiçoar a metodologia de treino, tendo em vista que a única literatura encontrada abordando a comparação entre técnicas em busca da melhora cognitiva, não trabalhou com grupo controle sem alteração em sua rotina diária. ${ }^{(20)}$ Resultados estatisticamente significativos foram encontrados na performance de memória após o treino mnemônico, e uma tendência para significância estatística no grupo de atividade física aeróbica, quando comparados ao grupo controle que apresentou uma diminuição discreta em sua capacidade de memorização.

Os resultados positivos para o treino de memória episódica no presente estudo podem estar relacionados, por exemplo, com o tipo de treino oferecido, isto é, foi ensinado somente um tipo de estratégia, a categorização. O ensino de estratégias de memória parece ser um processo gradual no qual inicialmente o participante não usa espontaneamente a estratégia (deficiência da produção); posteriormente passa a usá-la, mas o desempenho de memória não aumenta e; finalmente, o participante usa a estratégia com consequente aumento de seu desempenho. ${ }^{(27)}$

É possível que o treino intensivo com estratégia única possa ter favorecido a memória episódica, pois os idosos por apresentarem dificuldade em utilizar instantaneamente as estratégias de memória, necessitam de um espaço maior de tempo para assimilação das mesmas. É possível que o fato de não utilizarem automaticamente as estratégias recémadquiridas, possam exigir assim um maior consumo de recursos de processamento e, dessa maneira, não geram benefícios no desempenho propriamente dito. É plausível supor que treinos de memória com menos de quatro sessões não desenvolvam estratégias eficientes nos idosos, ${ }^{(28)}$ pois não foi oferecido aos mesmos o aprendizado de forma gradativa devido a limitação do tempo. 
Os resultados obtidos nesta pesquisa sugerem que os idosos participantes do treino intensificaram o uso da estratégia ensinada e, consequentemente, o desempenho de memória. Quanto aos resultados também positivos relacionados à atividade física aeróbia, porém não significativos estatisticamente acredita-se que no presente estudo não se tenha alcançado resultados estatisticamente significativos para o treinamento da atividade física aeróbica devido à limitação do reduzido número de sessões. Apesar de evidente melhora na comparação intra-grupo de atividade aeróbica próxima a significância estatística, sugere-se que futuras pesquisas sejam desenvolvidas durante um tempo mais longo para o treino, visto que o idoso, devido ao processo de envelhecimento, necessita de um tempo maior para apresentar melhoras fisiológicas mais expressivas advindas dos exercícios. É importante salientar que existem algumas limitações no presente estudo, tais como: 1) O MEEM pode não ser o instrumento mais indicado para uma avaliação cognitiva detalhada e, nesse sentido, pode causar um efeito falso-positivo dos sujeitos envolvidos nesse estudo; 2) O tamanho da amostra não permite estabelecer regra acerca da eficácia de uma técnica em detrimento de outra, 3) É importante salientar que nesse estudo foi utilizado um protocolo específico de treinamento de memória (técnica de categorização) e que outras formas de treinamento devem ser consideradas em futuros estudos; 4) O protocolo de atividade física aeróbia deve considerar variáveis para incrementar a intensidade do exercício, tais como: frequência cardíaca, dispneia, saturação de oxigênio dentre outras.

\section{CONCLUSÃO}

Conclui-se que o treinamento de memória foi mais eficaz em relação ao exercício físico (modalidade aeróbia) para o teste de figuras recordadas quando comparado os dados pré e pós - treinamento na amostra estudada.

O uso da atividade física como ferramenta para melhora da função cognitiva pode ser relevante, especialmente por sua aplicabilidade, pois se trata de um método relativamente barato que pode ser utilizado na população idosa.

\section{REFERÊNCIA}

1. Zambaldi PA, Costa TABNC, Diniz GCLM, Scalzo PL. Efeito de um treinamento de equilíbrio em um grupo de mulheres idosas da comunidade: estudo piloto de uma abordagem específica, não sistematizada e breve. Acta Fisiátrica. 2007;14(1):17-24. 
2. INSTITUTO BRASILEIRO DE GEOGRAFIA E ESTATÍSTICA (Brasil). Coordenação de população e indicadores sociais, projeções da população do Brasil por sexo e faixa etária. Revisão 2008. Rio de Janeiro: IBGE; 2010.

3. Ishizuka MA. Avaliação e comparação dos fatores intrínsecos dos riscos de quedas em idosos com diferentes estados funcionais [Dissertação]. São Paulo: Faculdade de Educação, Programa de Pós Graduação em Gerontologia da UNICAMP; 2003.

4. Kramer AF, Erickson KI. Capitalizing on cortical plasticity: influence of physical activity on cognition and brain function. Trends Cogn Sci. 2007;11(8):342-8.

5. Izquierdo I. Memória. $1^{\circ}$ ed. Porto Alegre: Artmed; 2002.

6. Wood GMO, Carvalho MRS, Neves RR, Haase VG. Validação da bateria de avaliação da memória de trabalho. Psicol Reflex Crít. 2001;14(2):325-41.

7. Charchat-Fichman H, Caramelli P, Sameshima K, Nitrini R. Declínio da capacidade cognitiva durante o envelhecimento. Rev Bras Psiquiatr. 2005 mar;27(12):79-82.

8. Yassuda MS, Lasca VB, Neri AL. Meta-memória e auto-eficácia: um estudo de validação de instrumentos de pesquisa sobre memória e envelhecimento. Psicol Reflex Crít. 2005 janabr;18(1):78-90.

9. Yassuda MS, Batistoni SST, Fortes AG, Neri AL. Treino de memória no idoso saudável: benefícios e mecanismos. Psicol Reflex Crít. 2006;19(3):470-481.

10. Erickson KI, Kramer AF. Aerobic exercise effects on cognitive and neural plasticity in older adults. Br J Sports Med. 2009 oct;43:22-4.

11. Yesavage JA, et al. Development and validation of a geriatric depression screening scale: A preliminary report. J Psychiatri Res. 1983;17(1):37-49.

12. Bertolucci PHF, Mathias SC, Brucki SMD, Carrilho, PEM . Proposta de padronização do mini-exame do estado mental (MEEM): estudo piloto cooperativo. Arq Neuropsiquiatr. 1994;52(1):225-40.

13. Banhato EFC, Scoralick NN, Guedes DV, Atalaia-Silva KC, Mota MMPE. Atividade física, cognição e envelhecimento: estudo de uma comunidade urbana. Psicol Teor Prát. 2009;11(1):76-84.

14.Paradela EMP, Lourenço RA, Veras RP. Validação da escala de depressão geriátrica em um ambulatório geral. Rev Saúde Pública. 2005;39(6):918-23.

15. Erzigkeit H. A short cognitive performance test for assessing deficits for memory and attention - SKT. Int Psychogeriatr. 1997;9(1):115-21.

16. Flaks MK, et al. The short cognitive performance test (SKT): preliminary study of its psychometric properties in Brazil. Int Psychogeriatr. 2006 mar;18(1):121-33. 
17. Pompéia S, Miranda MC, Bueno OFA. A set of 400 pictures standardised for portuguese. Arq Neuropsiquiatr. 2001;59(2-B):330-7.

18. Magalhães GM. Teste de caminhada dos seis minutos em uma população de idosos brasileiros [tese]. São Paulo: Universidade Cidade de São Paulo; 2008.

19. Verhaeghen P, Marcoen A, Goossens L. Improving memory performance in the aged through mnemonic training: A meta-analytic study. Psychol Aging. 1992 Jun;7(2):242-51.

20. Fabre C, Chamari K, Mucci P, Massé-Biron J, Préfaut C. Improvement of cognitive function by mental and/or individualized aerobic training in healthy elderly subjects. Int $\mathbf{J}$ Sports Med. 2002;23(6):415-21.

21. Silva VTBL, Souza MA, Pinheiro MHNP. Atividade física e os idosos do grupo São Vicente de Paula. Congresso Brasileiro de Geriatria e Gerontologia; 2004; Salvador.

Salvador: Interlinks Consultoria e Eventos; 2004.

22. De Vitta A. Atividade física e bem-estar na velhice. In: Neri AL, Freire SA. E por falar em boa velhice. Campinas: Papirus; 2000.p.81-9.

23. Studeski S, Carlson MC, Fillit H, Greenough WT, Kramer A, Rebok GW. From bedside to bench: does mental and physical activity promote cognitive vitality in late life? Sci Aging Knowledge Environ. 2006 jun;28(10):21.

24. Pietrelli A, Lopez-Costa J, Goñi R, Brusco A, Basso N. Aerobic exercise prevents agedependent cognitive decline and reduces anxiety-related behaviors in middle-aged and old rats. Neuroscience. 2012 jan;202(27):252-66.

25. Albeck DS, Sano K, Prewitt GE, Dalton L. Mild forced treadmill exercise enhances spatial learning in the aged rat. Behav Brain Res. 2006 apr;168(2):345-8.

26. Antunes KMH, Santos RF, Cassilhas R, Santos RVT, Bueno OFA, Mello MT. Exercício físico e função cognitiva: uma revisão. Rev Bras Med Esporte. 2006 mar/abr;12(2):108-14.

27. Bjorklund DF, Miller PH, Coyle TR, Slawinski JL. Instructing children to use memory strategies: Evidence of utilization deficiencies in memory training studies. Dev Rev. 1997;17:411-41.

28. Carvalho FCR. Treino de memória episódica com idosos normais [tese]. Campinas: Faculdade de Educação da UNICAMP; 2006.

\footnotetext{
i Trabalho realizado no Curso de Pós-Graduação Stricto Sensu em Ciências da Motricidade Humana Laboratório de Neuromotricidade e Performance Motora (LAMPEM), Universidade Castelo Branco, Rio de Janeiro - RJ, Brasil.
} 